ESAIM: PROCEEDINGS, October 2007, Vol.20, 72-82

Mohammed-Najib Benbourhim, Patrick Chenin, Abdelhak Hassouni \& Jean-Baptiste Hiriart-Urruty, Editors

\title{
PSEUDO-DIFFERENTIAL OPERATOR ASSOCIATED TO THE RADIAL BASIS FUNCTIONS UNDER TENSION.
}

\author{
A. Bouhamidi ${ }^{1}$
}

\begin{abstract}
Radial basis functions under tension (RBFT) depend on a positive parameter, incorporate the concept of spline with tension and provide a convenient way for the control of the behavior of the interpolating surface. The RBFT involve a function which is not complicated than exponential and may be easily coded. In this paper, we show that the RBFT, as like thin plate spline, may be associated to a differential operator in a Beppo-Levi space type. Both smoothing and interpolating problems by RBFT are studied.

Résumé. Les fonctions splines radiales sous tension dépendent d'un paramètre positif. Ces fonctions permettent d'incorporer un concept de tension pour toute dimension de l'espace. On montre dans ce papier que ces fonctions sont associées à un opérateur pseudo-différentiel dans un espace de type BeppoLevi et on étudie le problème d'interpolation et de lissage. Des examples numériques sont donnés pour illustrer ce type d'approximation.
\end{abstract}

\section{INTRODUCTION}

The radial basis functions under tension (RBFT) was introduced in [5]. The RBFT depend on a positive parameter and provide a convenient way of controlling the behavior of the interpolating surface. The aim of this paper is to give an explicit definition and construction of the associated native space of the RBFT, as a Beppo-Levi space type (see Theorem 2 and remark 2 in [5, p.141]). We show that the RBFT, as the thin plate splines, may be carried out from a (pseudo-) differential operator with a simple fundamental solution arising in the formulation of the RBFT. Furthermore, in this paper, we investigate both interpolating and smoothing problems.

The spline under tension was first introduced by Schweikert [22] as a mean of eliminating extraneous inflection points in curve fitting by the cubic spline. With the proper use of the tension parameter, a user is able to reduce the length of the interpolating curve and hence remove extraneous bumps. In the limit, the curve reduces to piecewise linear interpolant as the tension parameter becomes large. The problem of interpolation by spline with the concept of tension has many applications, for instance, in geology for terrain modelling $[15,17,23]$.

The thin plate splines under tension for two variables has been previously addressed by Franke and Nielson [11] and by Franke [12]. In [11] Franke and Nielson proposed a method of a construction of surfaces under tension based on a triangulation of the domain containing the scattered interpolating points. The second approach of Franke [12], for the construction of bivariate splines under tension, models a physical process of thin plate under tension. The approach of Franke [12], is approximately parallel that the development of Harder and Desmarais [14] for thin plate splines and based on experimental results. The basis function used by Franke [12],

\footnotetext{
1 L.M.P.A, Université du Littoral Côte d'Opale, 50 rue F. Buisson BP699, F-62228 Calais Cedex, France;

e-mail: A.Bouhamidi@lmpa.univ-littoral.fr
}

(C) EDP Sciences, SMAI 2007 
resulted from the equation of thin plate subject to lateral loads and mid-plane forces which may be written in the general form as $\Delta^{2} W=\frac{1}{D}\left(q+N_{x} \frac{\partial^{2} W}{\partial x^{2}}+N_{y} \frac{\partial^{2} W}{\partial y^{2}}-N_{x y} \frac{\partial^{2} W}{\partial x \partial y}\right)$ where $W$ is the lateral deflection, $q$ is the lateral load, $N_{x}, N_{y}$ and $N_{x y}$ are the mid-plane forces, and $D$ depends on the properties of the plate material. In order to simplify the previous equation, Franke considered a thin plate under a point load at the origin and set $N_{x} / D=N_{y} / D=\tau^{2}$ and $N_{x y}=0$, which gives an equation in the following form $\Delta^{2} W-\tau^{2} \Delta W=p$, where $p$ is the point load at the origin, namely $p(0)=q / d$ and $p=0$ elsewhere. Radial symmetry is assumed and by setting $V=\Delta W=\frac{1}{r} \frac{d}{d r}\left(r \frac{d W}{d r}\right)$, one may obtain the polar form of the last equation $\frac{d}{d r}\left(r \frac{d V}{d r}\right)-\tau^{2} r V=0$. The last equation is a modified Bessel equation of order zero, which has for convenient solution with respect to the boundary conditions, the function $-(2 \pi)^{-1} K_{0}(\tau r)$, where $K_{0}$ is a modified Bessel function of order zero. Finally, the basis function proposed by Franke [12] is $W_{\tau}(r)=-(2 \pi)^{-1} \int_{0}^{r} t^{-1} \int_{0}^{t} K_{0}(\tau s) d s d t+C$, where $C$ is a constant which may be equal to zero. In the terms of Franke [12], the lack of an elementary representation of the basis function is not a serious problem. Franke [12] proposed that the function can be approximated, either by numerical approximation of the integral formulation above, or by considering the function as solution of ordinary differential equation. By the assumption of point loads, the problem of interpolation of scattered data $\left(x_{k}, y_{k}\right) \in \mathbb{R}^{2}$ for $k=1, \ldots, N$ by thin plate spline under tension, as proposed by Franke [12], has the following representation $F(x, y)=\sum_{k=1}^{N} A_{k} W_{\tau}\left(\sqrt{\left(x-x_{k}\right)^{2}+\left(y-y_{k}\right)^{2}}\right)+a$.

A theoretical framework of the problem of thin plate splines with tension was presented in [2-4] in $d$ dimensional space $\mathbb{R}^{d}$. Let us now give a brief description of the spline under tension as was presented in [2-4]. Let $X\left(\mathbb{R}^{d}\right)$ be the space of distributions whose derivatives of order one and two are square integrable on $\mathbb{R}^{d}$,

$$
X\left(\mathbb{R}^{d}\right)=\left\{u \in \mathcal{D}^{\prime}\left(\mathbb{R}^{d}\right) \quad: \quad D^{\alpha} u \in L^{2}\left(\mathbb{R}^{d}\right) \text { for }|\alpha|=1,2\right\}
$$

where $\mathcal{D}^{\prime}\left(\mathbb{R}^{d}\right)$ is the space of distributions on $\mathbb{R}^{d}$. In the space $X\left(\mathbb{R}^{d}\right)$, we consider the semi-scalar product

$$
(u \mid v)_{X}=\sum_{|\alpha|=2} \frac{2}{\alpha !} \int_{\mathbb{R}^{d}} D^{\alpha} u(x) D^{\alpha} v(x) d x+\tau^{2} \sum_{|\alpha|=1} \int_{\mathbb{R}^{d}} D^{\alpha} u(x) D^{\alpha} v(x) d x
$$

with its associated semi-norm denoted by $|\cdot|_{X}$. Given any set of scattered points $x_{1}, \ldots, x_{N}$ in $\mathbb{R}^{d}$ and any set of real values $f_{1}, \ldots, f_{N}$, there is a unique function $s_{\tau} \in X\left(\mathbb{R}^{d}\right)$ minimizing the semi-norm $||$.$X subject to the$ interpolating constrains $s_{\tau}\left(x_{i}\right)=f_{i}$ for $i=1, \ldots N$. The function $s_{\tau}$ has explicitly the form

$$
s_{\tau}(x)=\sum_{i=1}^{N} \lambda_{i} E_{d, \tau}\left(x-x_{i}\right)+\lambda_{N+1}, \quad \text { with } \quad \sum_{i=1}^{N} \lambda_{i}=0
$$

where the function $E_{d, \tau}$ is a fundamental solution in $\mathbb{R}^{d}$ of the differential operator $D_{\tau}=\Delta^{2}-\tau^{2} \Delta$, namely $E_{d, \tau}$ is a tempered distribution in $\mathbb{R}^{d}$ satisfying $\Delta^{2} E_{d, \tau}-\tau^{2} \Delta E_{d, \tau}=\delta$, where $\delta$ is Dirac measure at the origin. A crucial point of this theory is of finding a fundamental solution of the operator $D_{\tau}$ in $\mathbb{R}^{d}$ which has a simple expression. For $d=1,2,3$, a fundamental solution of the operator $\Delta^{2}-\tau^{2} \Delta$ is given by (see $[3,4]$ )

$$
E_{d, \tau}(x)= \begin{cases}c_{1}\left(e^{-\tau|x|}+\tau|x|\right) & \text { for } d=1 \\ c_{2}\left(K_{0}(\tau|x|)+\ln (|x|)\right) & \text { for } d=2 \\ c_{3} \frac{e^{-\tau|x|}-1}{|x|} & \text { for } d=3\end{cases}
$$

where $K_{0}$ is the modified Bessel function and $c_{1}=-1 /\left(2 \tau^{3}\right), c_{2}=-1 /\left(2 \pi \tau^{2}\right)$ and $c_{3}=-1 /\left(4 \pi \tau^{2}\right)$. In the paper, we have used $|$.$| to denote the Euclidean norm in \mathbb{R}^{d}$.

The disadvantage of this representation is as follows. An explicit expression of the fundamental solution is, in general, unknown, for all $d \geq 4$, and the fundamental solution does not have an elementary explicit expression, for 
instance for $d=2$, its expression involves a Bessel function (which is more complicate than the usual functions). Furthermore, this fundamental solution does not have a similar explicit expression for all $d \geq 1$. The crucial question now is how to construct a (pseudo-)differential operator with a fundamental solution which generates a tempered distribution that has a simple and similar explicit expression for all dimension $d \geq 1$ and that allows the construction of the radial basis functions under tension as presented in [5]. The goal is the construction of a radial basis function depending on a tension parameter such that, as in the univariate case, in the limit the radial basis functions under tension reduce to the pseudo-linear splines as the tension parameter becomes large and reduce to the pseudo-cubic splines as the tension parameter becomes small. Let us now give here a brief recall of the theory of the pseudo-polynomial $(m, s)$-splines investigated by Duchon [10]. Let us consider the space $\widetilde{H}^{s}$ introduced by Peetre [19], which is the set of all tempered distributions $u$ whose Fourier transforms, denoted $\widehat{u}$ (or $\mathcal{F} u$ ), are locally integrable real-valued functions on $\mathbb{R}^{d}$ satisfying $\int_{\mathbb{R}^{d}}|\widehat{u}(\xi)|^{2}|\xi|^{2 s} d \xi<\infty$. Let $\mathcal{V}^{m, s}$ be the space of all distributions $u$ for which all the derivatives $D^{\alpha} u$ of order $|\alpha|=m$ are in $\widetilde{H}^{s}$. In the space $\mathcal{V}^{m, s}$ the following semi-norm

$$
\mathcal{N}_{m, s}(u)=\left(\sum_{|\alpha|=m} \frac{m !}{\alpha !} \int_{\mathbb{R}^{d}}\left|\widehat{D^{\alpha}} u(\xi)\right|^{2}|\xi|^{2 s} d \xi\right)^{1 / 2}
$$

is defined. Under the assumption $-m+\frac{d}{2}<s<\frac{d}{2}$, the space $\mathcal{V}^{m, s}$ with the semi-norm (4) is continuously embedded in the space $\mathcal{C}\left(\mathbb{R}^{d}\right)$ of continuous functions on $\mathbb{R}^{d}$. The pseudo-linear splines correspond to $s=s_{0}:=$ $\frac{d+1}{2}-m$ and $m \geq 1$, and the pseudo-cubic splines correspond to $s=s_{\infty}:=\frac{d+3}{2}-m$ and $m \geq 2$. We have $\mathcal{V}^{m, s_{\infty}} \hookrightarrow \mathcal{C}\left(\mathbb{R}^{d}\right)$ and $\mathcal{V}^{m, s_{0}} \hookrightarrow \mathcal{C}\left(\mathbb{R}^{d}\right)$.

Let $\mathcal{S}\left(\mathbb{R}^{d}\right)$ denote the space of rapidly decaying, infinitely differentiable functions on $\mathbb{R}^{d}$ and let $\mathcal{S}^{\prime}\left(\mathbb{R}^{d}\right)$ denote its dual space, the space of tempered distributions on $\mathbb{R}^{d}$. The space $\mathcal{D}\left(\mathbb{R}^{d}\right)=\mathcal{C}_{0}^{\infty}\left(\mathbb{R}^{d}\right)$ denotes the space of infinitely differentiable supported functions on $\mathbb{R}^{d}$.

\section{Associated Beppo-Levi space}

Let us introduce the following weight function

$$
\omega_{m, \tau}(\xi)=\tau^{2} \frac{|\xi|^{d+1-2 m}}{1-\left(\frac{|\xi|^{2}}{|\xi|^{2}+\tau^{2}}\right)^{\frac{d+1}{2}}},
$$

where $m \geq 1$ is a positive integer and $\tau>0$ is a positive parameter. We consider the following semi-norm

$$
|u|_{m, \tau}=\left(\sum_{|\alpha|=m} \frac{m !}{\alpha !} \int_{\mathbb{R}^{d}}\left|\widehat{D^{\alpha}} u(\xi)\right|^{2} \omega_{m, \tau}(\xi) d \xi\right)^{1 / 2},
$$

in an appropriate space $X_{\tau}^{m}\left(\mathbb{R}^{d}\right)$ (see below). The choose of the weight function given by (5), is motivated by the following arguments. By expanding the function $\omega_{m, \tau}$, we obtain the asymptotic behavior

$$
\omega_{m, \tau}(\xi) \sim_{\tau \rightarrow \infty} \tau^{2}|\xi|^{d+1-2 m} \text { a.e }
$$

namely, as $\tau \rightarrow \infty$, the weight function $\omega_{m, \tau}$ goes to the weight function associated to the pseudo-linear splines up to a multiplicative constant. And on the other hand, we have the asymptotic behavior

$$
\omega_{m, \tau}(\xi) \sim_{\tau \rightarrow 0} \frac{2}{d+1}|\xi|^{d+3-2 m} \text { a.e }
$$


which also means that, as $\tau \rightarrow 0$, the weight function $\omega_{m, \tau}$ goes to the weight function associated to the pseudocubic splines up to a multiplicative constant. Furthermore, the semi-norm $|\cdot|_{X}$ associated to the semi-scalar product (2) for curve spline under tension (see [2]) is given by

$$
|u|_{X}=\int_{-\infty}^{+\infty}\left|u^{\prime \prime}(t)\right|^{2} d t+\tau^{2} \int_{-\infty}^{+\infty}\left|u^{\prime}(t)\right|^{2} d t
$$

By using Plancherel's formula, the expression of the semi-norm (7) may be written in the following form

$$
|u|_{X}=\left(\int_{-\infty}^{+\infty}\left(|\xi|^{2}+\tau^{2}\right)\left|\widehat{u^{\prime}}(\xi)\right|^{2} d \xi\right)^{1 / 2}
$$

Observe that if we choose $m=1$ and $d=1$ in the expression (6), we obtain the semi-norm (8) minimizing by the curve spline under tension. We will show that the RBFT given in [5], may be derived from the minimization problem of the semi-norm (6) in an appropriate Beppo-levi space type $X_{\tau}^{m}\left(\mathbb{R}^{d}\right)$.

We remark that the weight function $\omega_{m, \tau}$ satisfies

$$
1 \leq \frac{1}{1-\left(\frac{|\xi|^{2}}{|\xi|^{2}+\tau^{2}}\right)^{\frac{d+1}{2}}} \leq \frac{1}{\tau^{2}}|\xi|^{2}+1, \forall \xi \in \mathbb{R}^{d}
$$

which gives

$$
\tau^{2}|\xi|^{d+1-2 m} \leq \omega_{m, \tau}(\xi) \leq|\xi|^{d+3-2 m}+\tau^{2}|\xi|^{d+1-2 m} .
$$

Let us consider the subspace $H_{\omega_{m, \tau}}$ of tempered distributions $u$, whose Fourier transforms $\widehat{u}$ are locally integrable real-valued functions on $\mathbb{R}^{d}$ satisfying $\int_{\mathbb{R}^{d}}|\widehat{u}(\xi)|^{2} \omega_{m, \tau}(\xi) d \xi<\infty$. On this space, we consider the following scalar product

$$
(u \mid v)_{\omega_{m, \tau}}=\int_{\mathbb{R}^{d}} \widehat{u}(\xi) \overline{\widehat{v}(\xi)} \omega_{m, \tau}(\xi) d \xi
$$

The associated norm is denoted by $\|u\|_{\omega_{m, \tau}}=(u \mid u)_{\omega_{m, \tau}}^{1 / 2}$.

Theorem 1. The space $H_{\omega_{m, \tau}}$ endowed with the scalar product (10) is a subspace of $\tilde{H}^{s_{0}}$, with continuous injection and it is a Hilbert subspace of $\mathcal{S}^{\prime}\left(\mathbb{R}^{d}\right)$ with continuous injection.

Proof. This is an immediate consequence of the inequality (9) and the properties of the space $\widetilde{H}^{s}$ of Peetre $[19]$.

Let us consider the functional Beppo-Levi type space [8], $X_{\tau}^{m}\left(\mathbb{R}^{d}\right)=B L_{m}\left(H_{\omega_{m, \tau}}\right)$ of distributions on $\mathbb{R}^{d}$ whose derivatives of order $|\alpha|=m$ are in $H_{\omega_{m, \tau}}$, namely

$$
X_{\tau}^{m}\left(\mathbb{R}^{d}\right)=\left\{u \in \mathcal{D}^{\prime}\left(\mathbb{R}^{d}\right) \quad ; \quad D^{\alpha} u \in H_{\omega_{m, \tau}}, \quad \forall \alpha \in \mathbb{N}^{d},|\alpha|=m\right\}
$$

A semi-inner product in $X_{\tau}^{m}\left(\mathbb{R}^{d}\right)$ is given by

$$
(u \mid v)_{m, \tau}=\sum_{|\alpha|=m} \frac{m !}{\alpha !} \int_{\mathbb{R}^{d}} \widehat{D^{\alpha}} u(\xi) \widehat{\widehat{D^{\alpha} v}(\xi)} \omega_{m, \tau}(\xi) d \xi, \quad \forall u, v \in X_{\tau}^{m}\left(\mathbb{R}^{d}\right) .
$$

The associated semi-norm is $|u|_{m, \tau}=(u \mid u)_{m, \tau}^{1 / 2}$. The null subspace of the semi-normed space $\left(X_{\tau}^{m}\left(\mathbb{R}^{d}\right),|\cdot|{ }_{m, \tau}\right)$ is $\Pi_{m-1}$ the space of polynomials of degree $\leq m-1$ on $\mathbb{R}^{d}$. 
Theorem 2. The space $X_{\tau}^{m}\left(\mathbb{R}^{d}\right)$ satisfies the following properties

a) $\mathcal{V}^{m, s_{\infty}} \cap \mathcal{V}^{m, s_{0}} \subset X_{\tau}^{m}\left(\mathbb{R}^{d}\right) \subset \mathcal{V}^{m, s_{0}}$ and the last inclusion is with continuous injection.

b) $\mathcal{S}\left(\mathbb{R}^{d}\right) \subset X_{\tau}^{m}\left(\mathbb{R}^{d}\right) \subset \mathcal{S}^{\prime}\left(\mathbb{R}^{d}\right)$.

c) The space $X_{\tau}^{m}\left(\mathbb{R}^{d}\right)$ is a semi-Hilbert subspace of the space $\mathcal{C}\left(\mathbb{R}^{d}\right)$ with continuous injection.

Proof. Let $u$ be a distribution such that for all multi-index $\alpha$ with $|\alpha|=m$, the Fourier transform $\widehat{D^{\alpha} u}$ of $D^{\alpha} u$ is a locally integrable function on $\mathbb{R}^{d}$. ¿From the inequality (9), we have

$$
\tau \mathcal{N}_{m, s_{0}}(u) \leq|u|_{m, \tau} \leq \sqrt{\mathcal{N}_{m, s_{\infty}}^{2}(u)+\tau^{2} \mathcal{N}_{m, s_{0}}^{2}(u)}
$$

The inequalities (13) prove the item a). The other items follow from the properties of the spaces $\mathcal{V}^{m, s_{0}}$ and $\mathcal{V}^{m, s_{\infty}}$.

\section{Associated Pseudo-Differential operator}

Let us define the pseudo-differential operator $\Delta_{d, \tau}: \mathcal{S}^{\prime}\left(\mathbb{R}^{d}\right) \rightarrow \mathcal{S}^{\prime}\left(\mathbb{R}^{d}\right)$ as a differential operator such that for a tempered distribution $T$, the Fourier transform of $\Delta_{d, \tau} T$ is a tempered distribution given by

$$
\widehat{\Delta_{d, \tau} T}=\omega_{m, \tau}(\xi)|\xi|^{2 m} \widehat{T}=\tau^{2} \frac{|\xi|^{d+1}}{1-\left(\frac{|\xi|^{2}}{|\xi|^{2}+\tau^{2}}\right)^{\frac{d+1}{2}}} \widehat{T}
$$

As usual in the distributions theory, we use the standard notation $\langle T, \varphi\rangle$, to denote the action of a distribution $T$ on a test function $\varphi$.

Proposition 1. a) For any element $u$ of $X_{\tau}^{m}\left(\mathbb{R}^{d}\right)$, the distribution $\Delta_{d, \tau} u$ is well-defined as a tempered distribution such that for any function $\varphi$ in $\mathcal{S}\left(\mathbb{R}^{d}\right)$,

$$
\left\langle\Delta_{d, \tau} u, \varphi\right\rangle=\left\langle u, \Delta_{d, \tau} \varphi\right\rangle=(u \mid \varphi)_{m, \tau} .
$$

b) If $u$ is an element of $X_{\tau}^{m}\left(\mathbb{R}^{d}\right)$ such that $\Delta_{d, \tau} u=0$, then $u$ is a polynomial in $\Pi_{m-1}$.

Proof. a) Let $u \in X_{\tau}^{m}\left(\mathbb{R}^{d}\right) \subset \mathcal{S}^{\prime}\left(\mathbb{R}^{d}\right)$ and let $\alpha$ be a multi-index such that $|\alpha|=m$. We have $g_{\alpha}:=(i \xi)^{2 \alpha} \omega_{m, \tau} \widehat{u}=$ $(i \xi)^{\alpha} \omega_{m, \tau} \widehat{D^{\alpha} u} \in L_{l o c}^{1}\left(\mathbb{R}^{d}\right)$ and $g_{\alpha}$ is a distribution on $\mathbb{R}^{d}$. Let $\left(\varphi_{n}\right)_{n}$ be a sequence of functions in $\mathcal{D}\left(\mathbb{R}^{d}\right)$ which converges to zero in $\mathcal{S}\left(\mathbb{R}^{d}\right)$. We have $\left\langle g_{\alpha}, \varphi_{n}\right\rangle=\int_{\mathbb{R}^{d}} \omega_{m, \tau}(\xi)(i \xi)^{\alpha} \widehat{D^{\alpha}} u(\xi) \varphi_{n}(\xi) d \xi$. By virtue of the CauchySchwarz's inequality, we have $\left|\left\langle g_{\alpha}, \varphi_{n}\right\rangle\right| \leq\left(\int_{\mathbb{R}^{d}} \omega_{m, \tau}(\xi)\left|\widehat{D^{\alpha}} u(\xi)\right|^{2} d \xi\right)^{1 / 2}\left(\int_{\mathbb{R}^{d}} \omega_{m, \tau}(\xi)\left|(i \xi)^{\alpha} \varphi_{n}(\xi)\right|^{2} d \xi\right)^{1 / 2}$. Since, $\left|(i \xi)^{\alpha}\right| \leq|\xi|^{|\alpha|}=|\xi|^{m}$, and according to $(9)$, we obtain

$$
\left|\left\langle g_{\alpha}, \varphi_{n}\right\rangle\right| \leq C \sup _{\xi \in \mathbb{R}^{d}}\left|\left(1+|\xi|^{2}\right)^{d+1} \varphi_{n}(\xi)\right|,
$$

where $0<C:=\max \left(1+\tau^{2}, 2\right)|u|_{m, \tau}\left[\int_{|\xi| \leq 1}|\xi|^{d+1} d \xi+\int_{|\xi|>1} \frac{1}{|\xi|^{3 d+1}} d \xi\right]^{1 / 2}<\infty$ is a positive constant. Since the sequence $\left(\varphi_{n}\right)_{n}$ converges to zero in $\mathcal{S}\left(\mathbb{R}^{d}\right)$, it follows from (14) that the sequence $\left\langle g_{\alpha}, \varphi_{n}\right\rangle$ converges to zero and this shows that the distribution $g_{\alpha}$ is tempered. Then, the linear combination

$$
\sum_{|\alpha|=m} \frac{m !}{\alpha !} g_{\alpha}=(-1)^{m} \omega_{m, \tau}(\xi)|\xi|^{2 m} \widehat{u}=(-1)^{m} \widehat{\Delta_{d, \tau} u}
$$

is also a tempered distribution and consequently, $\Delta_{d, \tau} u$ is well-defined as a tempered distribution. Furthermore, for any $\varphi \in \mathcal{S}$, we have $(u \mid \varphi)_{m, \tau}=\sum_{|\alpha|=m} \frac{m !}{\alpha !} \int_{\mathbb{R}^{d}} \xi^{2 \alpha} \omega_{m, \tau}(\xi) \widehat{u}(\xi) \widehat{\varphi}(\xi) d \xi$, and using the relation $\sum_{|\alpha|=m} \frac{m !}{\alpha !} \xi^{2 \alpha}=$ 
$|\xi|^{2 m}$, we obtain that $(u \mid \varphi)_{m, \tau}=\left\langle\mathcal{F}\left[\Delta_{d, \tau} u\right], \overline{\mathcal{F} \varphi}\right\rangle=\left\langle\mathcal{F} u, \overline{\mathcal{F}\left[\Delta_{d, \tau} \varphi\right]}\right\rangle$. Since for any distribution $T \in \mathcal{S}^{\prime}\left(\mathbb{R}^{d}\right)$ and any function $\varphi \in \mathcal{S}\left(\mathbb{R}^{d}\right)$, we have the relation $\langle\mathcal{F} T, \overline{\mathcal{F}} \varphi\rangle=\langle T, \varphi\rangle$, the result follows.

$b)$ Let $u$ be an element of $X_{\tau}^{m}\left(\mathbb{R}^{d}\right)$, such that $\Delta_{d, \tau} u=0$, it follows that $\omega_{m, \tau}(\xi)|\xi|^{2 m} \widehat{u}=0$, then $|\xi|^{d+1} \widehat{u}=0$. The function $\xi \mapsto|\xi|^{d+1}$ vanishes only at the origin. It follows that $\widehat{u}$ is a distribution whose support is the origin. From Schwartz's Theorem [20, p.100], $\widehat{u}$ is a finite linear combination of Dirac's measure at the origin and its derivatives. Hence $u$ is a polynomial, and its derivatives $D^{\alpha} u$ for all $|\alpha|=m$ are also polynomials belonging to $H_{\omega_{m, \tau}}$. But the only polynomial in $H_{\omega_{m, \tau}}$ is the zero polynomial, thus $D^{\alpha} u=0$ for $|\alpha|=m$, namely $u$ is in $\Pi_{m-1}$.

A fundamental solution of the differential operator $\Delta_{d, \tau}$ is a tempered distribution $\Phi_{d, \tau}$ such that

$$
\Delta_{d, \tau} \Phi_{d, \tau}=\delta
$$

where $\delta$ is Dirac's measure at the origin. The Fourier transform $\widehat{\Phi}_{d, \tau}$ of $\Phi_{d, \tau}$ satisfies the following relation

$$
\omega_{m, \tau}(\xi)|\xi|^{2 m} \widehat{\Phi}_{d, \tau}=1
$$

Theorem 3. A fundamental solution of the operator $\Delta_{d, \tau}$ is the tempered distribution $\Phi_{d, \tau}$ induced by the function, also denoted $\Phi_{d, \tau}$ and given by

$$
\Phi_{d, \tau}(x)=C_{d, \tau}\left(e^{-\tau|x|}+\tau|x|\right), \quad \text { where } C_{d, \tau}=-\frac{1}{2^{d} \tau^{3} \pi^{(d-1) / 2} \Gamma\left(\frac{d+1}{2}\right)},
$$

where $\Gamma$ is the Gamma function. The function $\Phi_{d, \tau}$ has a continuous extension to a function of class $\mathcal{C}^{2}$ on $\mathbb{R}^{d}$.

Proof. Let $\Phi_{1}(x)=\tau|x|$ and $\Phi_{2}(x)=e^{-\tau|x|}$. The distributions induced by the functions $\Phi_{1}$ and $\Phi_{2}$ and also denoted by $\Phi_{1}$ and $\Phi_{2}$, respectively, are tempered. We recall the following two formulas, which are accessible in the literature. The first one (see, for instance, [13, pp 172-173])

$$
\widehat{|\xi|^{\alpha}}=2^{d+\alpha} \pi^{d / 2} \frac{\Gamma\left(\frac{d+\alpha}{2}\right)}{\Gamma\left(-\frac{\alpha}{2}\right)} F_{p}\left[\frac{1}{|\xi|^{d+\alpha}}\right]
$$

for $\alpha>0$ and $\alpha \notin 2 \mathbb{N}$, and the second one (see, for instance, [21, pp 163-165])

$$
\int_{0}^{+\infty} e^{-\beta t} J_{\nu}(\alpha t) t^{\nu+1} d t=2 \beta \frac{1}{\sqrt{\pi}}(2 \alpha)^{\nu} \Gamma\left(\nu+\frac{3}{2}\right)\left(\alpha^{2}+\beta^{2}\right)^{-\nu-\frac{3}{2}}
$$

where $\nu>-1, \beta>0, \alpha \in \mathbb{R}$, and $J_{\nu}$ is a Bessel function. From formula (18), for $\alpha=1$, we get the Fourier transform of the distribution $\Phi_{1}$ as $\widehat{\Phi}_{1}=-2^{d} \tau \pi^{(d-1) / 2} \Gamma\left(\frac{d+1}{2}\right) F_{p}\left[\frac{1}{|\xi|^{d+1}}\right]$. Thus

$$
\omega_{m, \tau}(\xi)|\xi|^{2 m} \widehat{\Phi}_{1}=-2^{d} \tau \pi^{(d-1) / 2} \Gamma\left(\frac{d+1}{2}\right) \omega_{m, \tau}(\xi) F_{p}\left[\frac{1}{|\xi|^{d+1-2 m}}\right] .
$$

Since $\xi \mapsto \frac{1}{|\xi|^{d+1-2 m}}$ is in fact a locally integrable function, thus the symbol $F_{p}$ for finite part of the distribution is useless. Now, from formula (19), for $\beta=\tau, \alpha=|\xi|$ and $\nu=\frac{d-2}{2}$ we get the following formula

$$
(2 \pi)^{d / 2}|\xi|^{\frac{2-d}{2}} \int_{0}^{+\infty} e^{-\tau t} J_{\frac{d-2}{2}}(|\xi| t) t^{\frac{d}{2}} d t=2^{d} \tau \pi^{(d-1) / 2} \Gamma\left(\frac{d+1}{2}\right)\left(|\xi|^{2}+\tau^{2}\right)^{-\frac{d+1}{2}}
$$


which means by the Hankel transform that the Fourier transform of the function $\Phi_{2}$ is given by $\widehat{\Phi}_{2}=$ $2^{d} \tau \pi^{(d-1) / 2} \Gamma\left(\frac{d+1}{2}\right)\left(|\xi|^{2}+\tau^{2}\right)^{-\frac{d+1}{2}}$. Thus

$$
\omega_{m, \tau}(\xi)|\xi|^{2 m} \widehat{\Phi}_{2}=2^{d} \tau \pi^{(d-1) / 2} \Gamma\left(\frac{d+1}{2}\right) \omega_{m, \tau}(\xi)|\xi|^{2 m}\left(|\xi|^{2}+\tau^{2}\right)^{-\frac{d+1}{2}} .
$$

Let $A_{d, \tau}=-2^{d} \tau \pi^{(d-1) / 2} \Gamma\left(\frac{d+1}{2}\right)$. From relations (20) and (21), we get $\omega_{m, \tau}(\xi)|\xi|^{2 m} \mathcal{F}\left[\Phi_{1}+\Phi_{2}\right]=\tau^{2} A_{d, \tau}$, which means that the Fourier transform of the function $\Phi_{d, \tau}$ satisfies $\omega_{m, \tau}(\xi)|\xi|^{2 m} \widehat{\Phi}_{d, \tau}=1$, namely, $\widehat{\Delta_{d, \tau} \Phi_{d, \tau}}=1=\widehat{\delta}$. Therefore $\Delta_{d, \tau} \Phi_{d, \tau}=\delta$, and $\Phi_{d, \tau}$ is a fundamental solution of the pseudo-differential operator $\Delta_{d, \tau}$.

It is obvious to check that the function $\Phi_{d, \tau}$ has a continuous extension to a function of class $\mathcal{C}^{2}$ on $\mathbb{R}^{d}$, which still denoted $\Phi_{d, \tau}$ with the extension to zero given, for $i, j=1, \cdots, d$, by $\frac{\partial \Phi_{d, \tau}}{\partial x_{i}}(0)=0, \frac{\partial^{2} \Phi_{d, \tau}}{\partial x_{i} \partial x_{j}}(0)=0$ for $i \neq j$ and $\frac{\partial^{2} \Phi_{d, \tau}}{\partial x_{i}^{2}}(0)=-C_{d, \tau} \tau^{2}=\frac{1}{2^{d} \tau \pi^{(d-1) / 2} \Gamma\left(\frac{d+1}{2}\right)}$.

A compactly supported measure $\mu$ on $\mathbb{R}^{d}$, is said to be orthogonal to $\Pi_{m-1}$, if and only if it satisfies the orthogonal condition $\int_{\mathbb{R}^{d}} \xi^{\alpha} d \mu(\xi)=0$, for all multi-index $\alpha$ such that $|\alpha| \leq m-1$. This condition is equivalent to $D^{\beta} \widehat{\mu}(0)=0$, for all multi-index such that $|\alpha| \leq m-1$, where $\widehat{\mu}$ is the Fourier transform of the measure $\mu$. It is well known that the Fourier transform $\widehat{\mu}$ of a compactly supported measure $\mu$ on $\mathbb{R}^{d}$ is a slowly increasing bounded $\mathcal{C}^{\infty}$ function on $\mathbb{R}^{d}$.

Theorem 4. Let $\mu$ be a compactly supported measure, orthogonal to $\Pi_{m-1}$. Then

a) The convolution product $\mu * \Phi_{d, \tau}$ is an element of $X_{\tau}^{m}\left(\mathbb{R}^{d}\right)$.

b) We have $\Delta_{d, \tau}^{m}\left(\mu * \Phi_{d, \tau}\right)=\mu$.

c) For any element $u$ of $X_{\tau}^{m}\left(\mathbb{R}^{d}\right)$ we have $\langle\mu, u\rangle=\left(\mu * \Phi_{d, \tau} \mid u\right)_{m, \tau}$.

Proof. See [6].

It immediately follows from the item $c$ ) of Theorem 4 that for all compactly supported measure $\mu$ orthogonal to $\Pi_{m-1}$,

$$
\left\langle\mu, \mu * \Phi_{d, \tau}\right\rangle=\left(\mu * \Phi_{d, \tau} \mid \mu * \Phi_{d, \tau}\right)_{m, \tau}=\left|\mu * \Phi_{d, \tau}\right|_{m, \tau}^{2}>0
$$

Especially, for all measure $\mu=\sum_{k=1}^{p} c_{k} \delta_{x_{k}}$ supported at by finite set $\left\{x_{1}, \cdots, x_{p}\right\}$ of $p$ points of $\mathbb{R}^{d}$ such that $\langle\mu, p\rangle=\sum_{k=1}^{p} c_{k} p\left(x_{k}\right)=0$, for all $p \in \Pi_{m-1}$, we have $\left\langle\mu, \mu * \Phi_{d, \tau}\right\rangle=\sum_{i, j=1}^{p} c_{i} c_{j} \Phi_{d, \tau}\left(x_{i}-x_{j}\right)>0$, which means that the function $\Phi_{d, \tau}$ is strictly conditionally positive definite function of order $m \geq 1$ for all $d \geq 1$. For the definition of a strictly conditionally positive definite function see, for instance, $[7,18]$.

\section{VAriational Problem}

Let us give an arbitrary finite set $\mathcal{A}=\left\{x_{1}, \cdots, x_{N}\right\} \subset \mathbb{R}^{d}$ of scattered data points $\left(x_{i} \neq x_{j}\right)$. We assume that $N \geq d(m):=\operatorname{dim}\left(\Pi_{m-1}\right)$ and that $\mathcal{A}$ contains a $\Pi_{m-1}$-unisolvent subset, which means that if $p \in \Pi_{m-1}$ is such that $p\left(x_{i}\right)=0$ for $i=1, \cdots, d(m)$, then $p$ is the zero polynomial. Let $z=\left(z_{1}, \cdots, z_{N}\right)^{T}$ be a given vector in $\mathbb{R}^{N}$.

Consider the following interpolating problem

$$
\begin{aligned}
& \text { Find } u \in X_{\tau}^{m}\left(\mathbb{R}^{d}\right) \quad \text { such that } \\
& |u|_{m, \tau}=\min _{\substack{v \in X_{\tau}\left(\mathbb{R}^{d}\right) \\
v\left(x_{i}\right)=z_{i} \\
i=1, \cdots, N}}|v|_{m, \tau}
\end{aligned}
$$


and the smoothing problem

$$
\begin{aligned}
& \text { Find } u \in X_{\tau}^{m}\left(\mathbb{R}^{d}\right) \text { such that } \\
& J_{\rho}(u)=\min _{v \in X_{\tau}^{m}\left(\mathbb{R}^{d}\right)} J_{\rho}(v),
\end{aligned}
$$

where

$$
J_{\rho}(v)=|v|_{m, \tau}^{2}+\frac{1}{\rho} \sum_{i=1}^{N}\left(v\left(x_{i}\right)-z_{i}\right)^{2} .
$$

In the space $X_{\tau}^{m}\left(\mathbb{R}^{d}\right)$, we consider the scalar product given by

$$
\langle u \mid v\rangle_{m, \tau}=(u \mid v)_{m, \tau}+\frac{1}{\varepsilon} \sum_{i=1}^{N} u\left(x_{i}\right) v\left(x_{i}\right)
$$

where the parameter $\varepsilon=1$ for the interpolating problem and $\varepsilon=\rho$ for the smoothing problem, is fixed once and for all the rest of this Section. Let $\|u\|_{m, \tau}$ the associated norm of the scalar product given by (24).

The following proposition is an immediate consequence of Theorem 2,

Proposition 2. The space $X_{\tau}^{m}\left(\mathbb{R}^{d}\right)$ endowed with the scalar product (24) and its associated norm is a Hilbert subspace of $\mathcal{C}(\mathbb{R})$ with continuous injection.

Now, we can state the following Theorem

Theorem 5. Let $N$ be an integer such that $N \geq d(m)$, let $\mathcal{A}=\left\{x_{1}, \cdots, x_{N}\right\}$ be a finite set of distinct points in $\mathbb{R}^{d}$ which contains a $\Pi_{m-1}$-unisolvent subset and let $z=\left(z_{1}, \cdots, z_{N}\right)^{T} \in \mathbb{R}^{N}$. Then, the interpolating problem (22) and the smoothing problem $(23)$ have a unique solution $\sigma_{\tau}^{\mathcal{A}}$ and $\sigma_{\tau, \rho}^{\mathcal{A}}$ in $X_{\tau}^{m}\left(\mathbb{R}^{d}\right)$, respectively. The solutions are explicitly given by

and

$$
\sigma_{\tau}^{\mathcal{A}}(x)=\sum_{i=1}^{N} \lambda_{i} \Phi_{d, \tau}\left(x-x_{i}\right)+\sum_{i=1}^{d(m)} \alpha_{i} p_{i}(x),
$$

$$
\sigma_{\tau, \rho}^{\mathcal{A}}(x)=\sum_{i=1}^{N} \mu_{i} \Phi_{d, \tau}\left(x-x_{i}\right)+\sum_{i=1}^{d(m)} \beta_{i} p_{i}(x),
$$

where $\Phi_{d, \tau}$ is given by (17) and $\left(p_{j}\right)_{j=1, \cdots, d(m)}$ is a basis of $\Pi_{m-1}$. The vectors $\lambda=\left(\lambda_{1}, \cdots, \lambda_{N}\right)^{T}, \mu=$ $\left(\mu_{1}, \cdots, \mu_{N}\right)^{T}, \alpha=\left(\alpha_{1}, \cdots, \alpha_{d(m)}\right)^{T}$ and $\beta=\left(\beta_{1}, \cdots, \beta_{d(m)}\right)^{T}$ are computed by solving the following nonsingular linear systems

$$
\left(\begin{array}{cc}
A & M \\
M^{T} & O
\end{array}\right)\left(\begin{array}{l}
\lambda \\
\alpha
\end{array}\right)=\left(\begin{array}{c}
z \\
O
\end{array}\right), \text { and }\left(\begin{array}{cc}
A+\rho I_{N} & M \\
M^{T} & O
\end{array}\right)\left(\begin{array}{c}
\mu \\
\beta
\end{array}\right)=\left(\begin{array}{c}
z \\
O
\end{array}\right)
$$

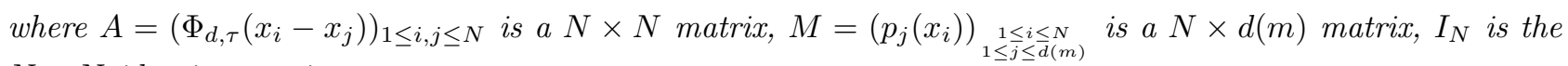
$N \times N$ identity matrix.

Proof. The proof of this theorem is based on the classical abstract theory of spline functions. For instance, Theorem 4.4.2 in [16, p. 196] and Theorem 4.4.4 in [16, p. 201] prove the result (see also Theorem 1.1 in [1, p. 147] and Theorem 2.1 in [1, p. 153]). The fact that the linear systems are nonsingular resulted from the conditionally positive definiteness of the function $\Phi_{d, \tau}$ (see, for instance, $[7,18]$ )

\section{Numerical RESults}

In this section, we give some examples of approximations by radial basis function with tension. All our computations were carried out using Matlab 6.5 on an Intel Pentium workstation with about 16 significant decimal digits. 


\subsection{Example 1}

In the first example we consider the problem of interpolation in one variable. The function to be interpolated is the function $f(x)=\frac{1}{2}(10+\tanh (x))$ restricted to the range $[-5,5]$. We interpolate the function at the following $N=7$ points $x_{1}=-5, x_{2}=-2, x_{3}=-0.5, x_{4}=0, x_{5}=1, x_{6}=2$ and $x_{7}=5$ by a cubic spline and by the RBFT with $\tau=0.91$. Figure 1 shows the results. We observe that the cubic spline presents some extraneus values relative to the interpolating function. This problem is eliminated by using RBFT. The relative error of the cubic spline to the original function is numerically computed and is $1.39 e-002$. Since the relative error of the RBFT with $\tau=0.91$ to the original function is $8.28 e-004$.
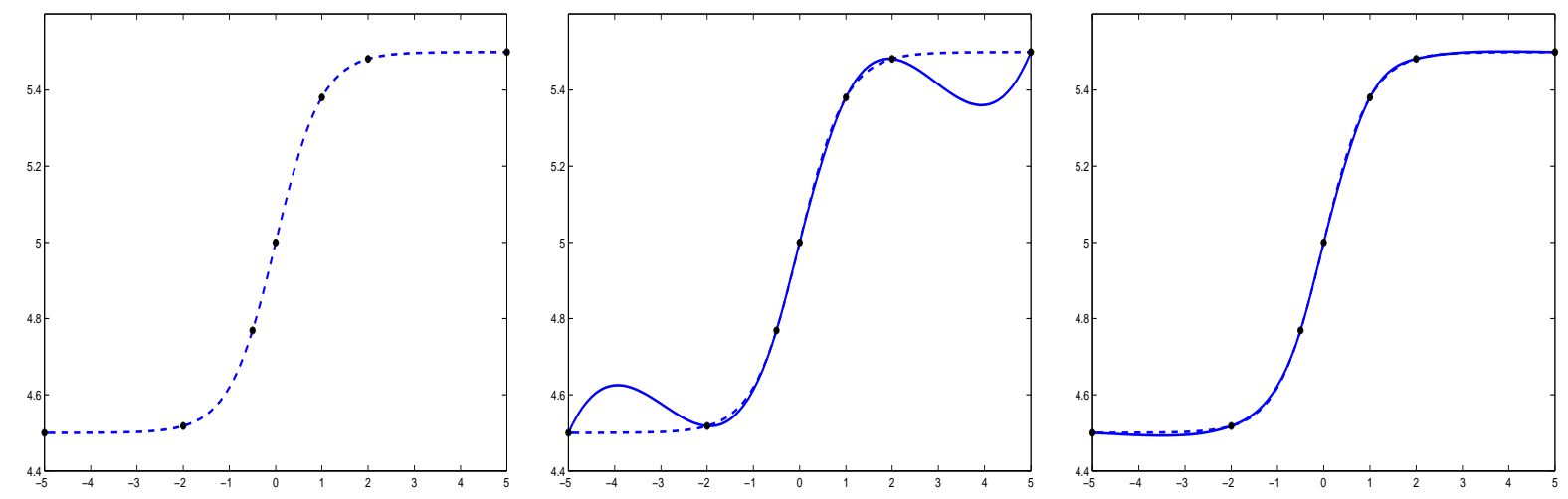

FIgURE 1. The original function (left), the original function with the interpolating cubic spline (center), the original function with the RBFT with $\tau=0.91$ (right).

\subsection{Example 2}

In this example we consider the smoothing problem also in one variable with the original function $f$ is the one given in Example 1 . The $N=150$ points $x_{1}, \cdots, x_{N}$ are given in the range [-5,5] and we consider the values

$$
z_{i}=f\left(x_{i}\right)+\varepsilon_{i}, \quad i=1, \ldots, N
$$

where the $\varepsilon_{i}$ 's are independently, normally and identically distributed random variables. The function is approximated by using the smoothing RBFT for different values of the parameter $\tau$ of tension together with the values of the parameter $\rho$. We compared the method of the smoothing RBFT to the smoothing cubique spline. Figure 2 shows the results. The relative error of the smoothing cubic spline for $\rho=0.1$ to the original function is 0.0098 . Since the relative error of the $\operatorname{RBFT}$ for $\rho=0.1$ and $\tau=1$ is 0.0105 and for $\rho=0.1$ and $\tau=7$ is 0.0034 .

\subsection{Example 3}

The third example examines the case of interpolation of surface with two variables. It shows how the use of RBFT may help to avoid an undesirable extraneous overshoot of the interpolating surface. The function to be interpolated is given on the rectangle $[0,1] \times[0,1]$ by

$$
f(x, y)=\left\{\begin{array}{lll}
\frac{1}{2} & \text { if } \quad y \leq \frac{2}{5} \\
\frac{1}{2}\left(1-\frac{25}{9}\left(y-\frac{2}{5}\right)^{2}\right) & \text { if } \quad y>\frac{2}{5} \text { and } x \leq \frac{1}{5} \\
\frac{125}{72}(1-y)^{2}(1-x) & \text { if } & y>\frac{2}{5}
\end{array} \text { and } x>\frac{1}{5} .\right.
$$



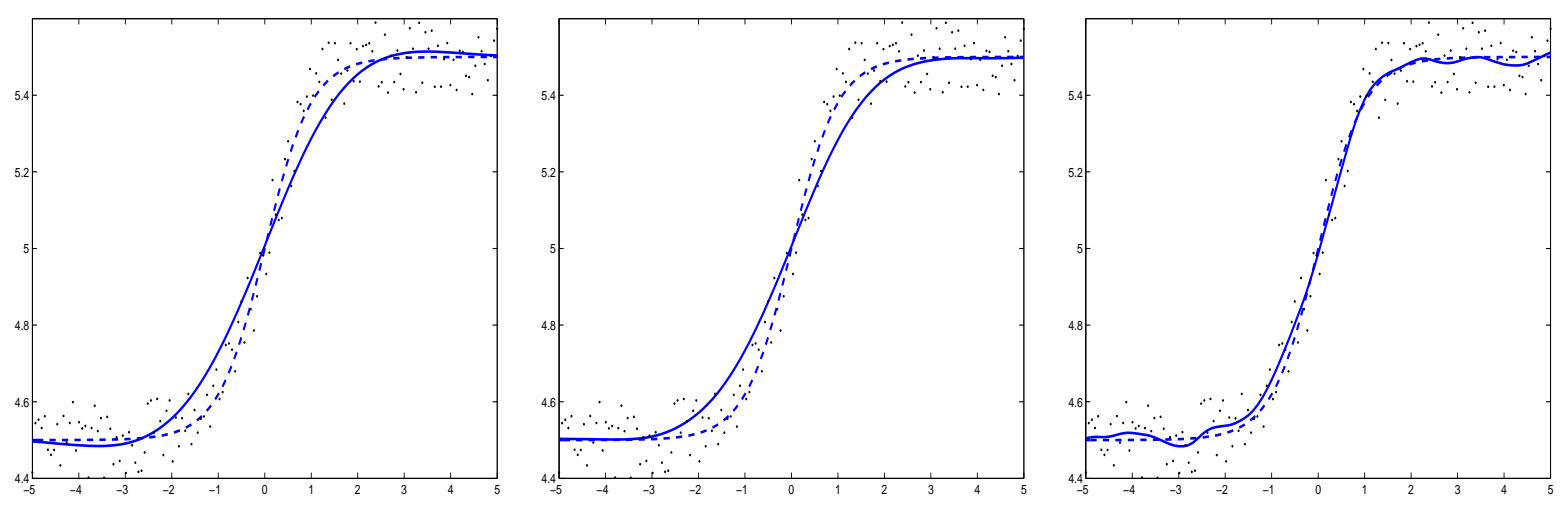

Figure 2. The original function with the smoothing cubic spline with $\rho=0.1$ (left), the original function with the the smoothing $\operatorname{RBFT}$ with $\tau=1$ and $\rho=0.1$ (center), the original function with the the smoothing $\operatorname{RBFT}$ with $\tau=6$ and $\rho=0.1$ (right).
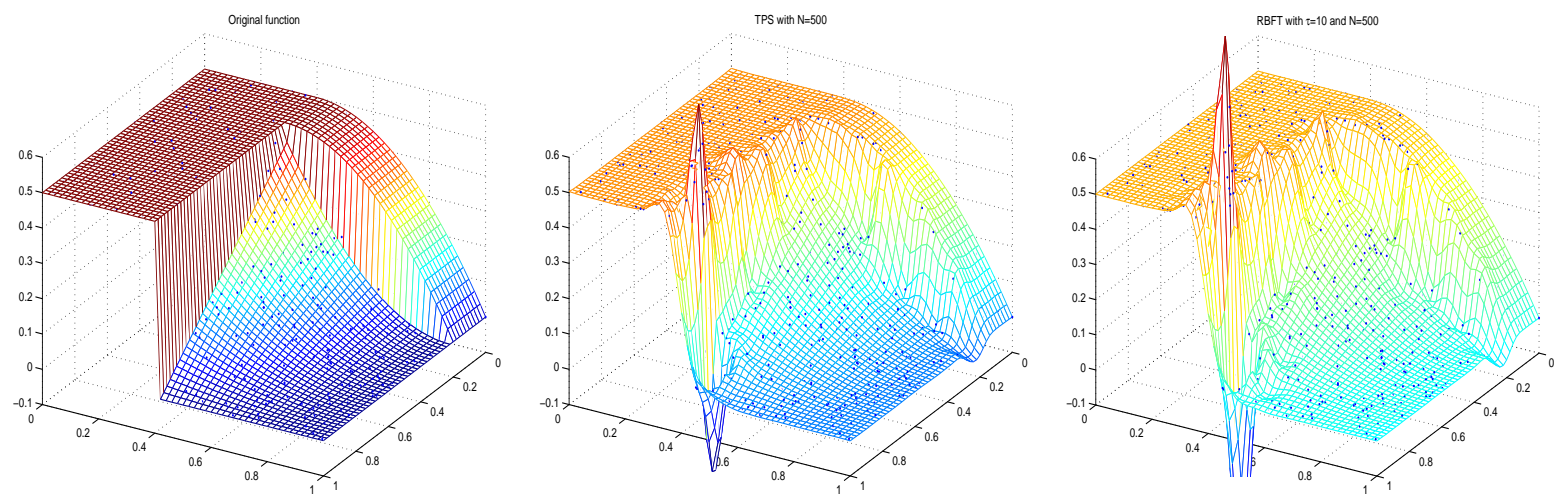

Figure 3. The original function (left), the TPS (center), the RBFT with $\tau=10$ (right). The number of points is $N=500$.

This is an extreme example since the interpolated original surface is discontinuous. Such surface models a terrain with a faults. We interpolated the function $f$ on a set of $N=500$ scattered data points randomly distributed on $[0,1] \times[0,1]$. Figure 3 shows the original function together with the interpolating thin plate spline and RBFT with $\tau=10$. we observe that the thin plate spline together with RBFT with small value of the parameter of tension present some overshoot. Figure 4 shows the RBFT for some increasing values of the parameter of tension, we observe that a large values of the the parameter of tension improve the result and illuminate the overshoot. 

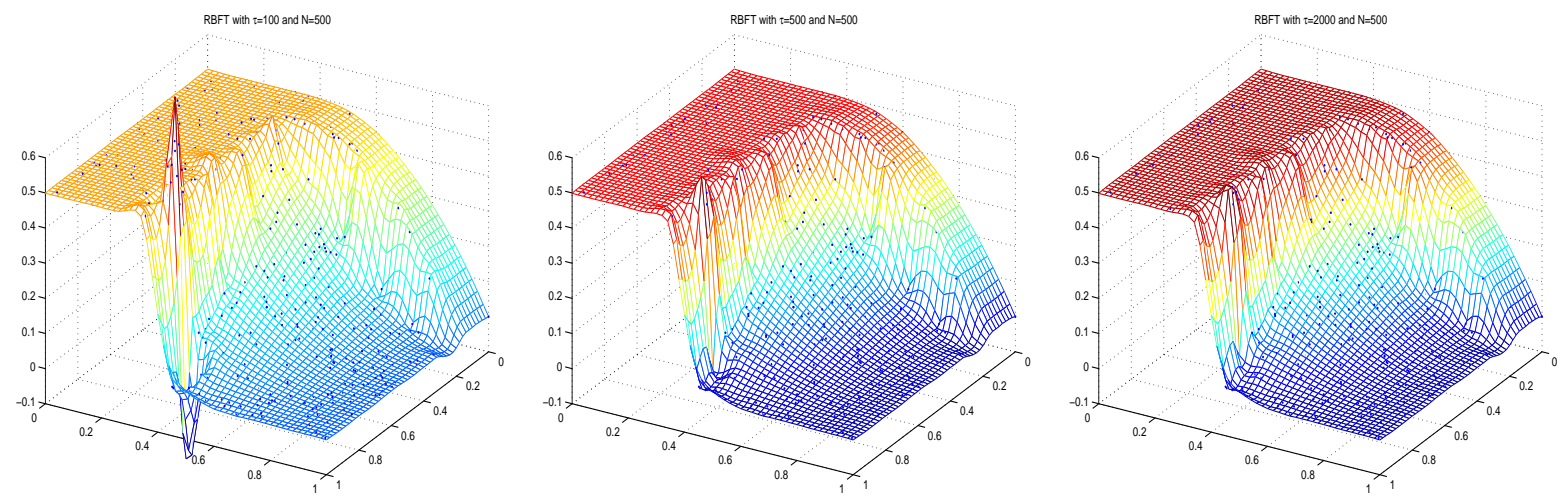

Figure 4. The RBFT with $\tau=100$ (left), the RBFT with $\tau=500$ (center), the RBFT with $\tau=2000$ (right). The number of points is $N=500$.

\section{REFERENCES}

[1] M. Attéia, Hilbertian Kernels and spline functions, Studies in Computational Mathematics, Vol.4, North-Holland, Amsterdam, 1992.

[2] A. Bouhamidi, Hilbertian approach for univariate spline with tension, Approx. Theory and its Appl. 17:4, (2001), pp. 36-57.

[3] A. Bouhamidi, A. Le Méhauté, Spline Curves and Surfaces with tension. Wavelets, Images, and Surface Fitting, (P. J. Laurent, A. Le Méhauté and L. L. Schumaker, Eds), A, K Peters Wellesley (1994), pp. 51-58.

[4] A. Bouhamidi, A. Le Méhauté, Multivariate Interpolating $(m, \ell, s)$-splines, Advances in Computational Mathematics. 11 (1999), pp. 287-314.

[5] A. Bouhamidi, A. Le Méhauté, Radial basis functions under tension, Journal of Approximation Theory 127 (2004), pp. 135-154.

[6] A. Bouhamidi, Weighted Thin plate splines, Analysis and Applications, Vol 2, No. 3 (2005), 297-324.

[7] M. D. Buhmann, Radial Basis functions, Cambridge Monographs on applied and computational mathematics, Cambridge University press (2003).

[8] J. Deny, J. L. Lions, Les espaces du type de Beppo-Levi, Ann. Inst. Fourier 5 (1954), pp. 305-370.

[9] J. Duchon, Interpolation de fonctions de deux variables suivant le principe de la flexion des plaques minces, R.A.I.R.O, Analyse numérique, Vol 10 (1975), pp. 5-12.

[10] J. Duchon, Splines minimizing rotation-invariant seminorms in Sobolev spaces, In Constructive Theory of Functions of Several Variables, Lecture Notes in Mathematics 571, eds. W. Schempp and K. Zeller, Springer-Verlag (Berlin), (1977), pp. 85-100.

[11] G. M. Nielson, R. Franke, A method for construction of surfaces under tension, Rocky Moutain J. Math. 14 (1984) $203-221$.

[12] R. Franke, Thin plate spline with tension, Comput. Aided Geom. Design 2 (1985),pp. 87-95.

[13] I. M. Gelfand, N. Ya Vilenkin, Generalized functions, vol 4, Academic Press, New York, 1964.

[14] R. L. Harder, R. N. Desmarais, Interpolation using surfaces splines, J. Aircraft 92 (1972),pp. 189-191.

[15] J. Hofierka, J. Parajka, H. Mitasova, L. Mitas, Multivariate interpolation of precipitation using regularized spline with tension Transactions in GIS, (2002), 135-150.

[16] P. J. Laurent, Approximation et optimisation. Hermann, Paris, 1972.

[17] H. Mitasova, L. Mitas, Interpolation by regularized spline with tension, I. Theory and implementation, II. Application to terrain modelling and surface geometry analysis. Mathematical Geology, 25, (1993),641-655 and 657-669.

[18] C. A. Michelli, Interpolation of scattered data: Distance matrices and conditionally positive definte functions, Constr. Approx. 2 (1986), pp. 11-22.

[19] J. Peetre, Espaces d'interpolation et théorème de Sobolev, Ann. Inst. Fourier, Grenoble, 16, (1966), pp. $279-317$.

[20] L. Schwartz, Théorie des distributions, Hermann, Paris (1966).

[21] L. Schwartz, Analyse IV, Applications à la théorie de la mesure, Hermann, Paris (1993, second edition).

[22] D. G. Schweikert, An interpolation curve using a spline in tension. J. Math. and Phys, vol 45,(1966), 312-317.

[23] P. Wessel, D. Bercovici, Gridding with spline in tension: A Green functions Approach, Maths Geology., 30, (1998), 77-93. 